\title{
229 CX3CR1 IN EXHAUSTED CD8 T CELL STATES
}

${ }^{1}$ Apoorvi Chaudhri*, ${ }^{2}$ Yunfei Wang, ${ }^{3}$ Shao-Hsi Hung, ${ }^{3}$ Gregory Lizee, ${ }^{4}$ Ulrich Von Andrian,

${ }^{2}$ Patrick Hwu, ${ }^{5}$ Gordon Freeman. ${ }^{1}$ Dana-Farber Cancer Institute, Boston, MA, USA; MD Anderson Cancer Center, Houston, TX, USA; ${ }^{2}$ Moffitt Cancer Center, Tampa, FL, USA; ${ }^{3} M D$ Anderson Cancer Center, Houston, TX, USA; ${ }^{4}$ Harvard Medical School, Boston, MA, USA; ${ }^{5}$ Dana-Farber Cancer Institute, Boston, MA, USA

Background Cancer has chronic antigen exposure that results in a suppressed CD8 $\mathrm{T}$ cell state termed exhaustion. An outcome of anti PD-1 blockade therapy is the expansion of early exhausted CD8 $+\mathrm{T}$ cells into a terminally differentiated exhausted state. The reversal of this transcriptionally plastic yet epigenetically fixed state of CD8 $\mathrm{T}$ cell exhaustion has the potential to increase responses to anti PD-1 therapy.

Methods CX3CR1 is a marker of CD8 T cell activation, effector function however less is known about the contribution of CX3CR1 in CD8 $\mathrm{T}$ cell exhaustion. We identified three distinct subsets of CD8 + tumor infiltrating lymphocytes (TILs) based on high, mid, and negative CX3CR1 expression in a mouse model of colon carcinoma.

Results The CX3CR1 high CD8 $+\mathrm{T}$ cells are more exhausted with higher PD1+TIM3 + expression compared to CX3CR1 mid and CX3CR1 negative cells thereby representing the terminal state of CD8 T cell exhaustion. Moreover, CX3CR1 high CD8 $\mathrm{T}$ cells increase following anti PD-1 blockade, and their abundance is associated with a positive response to anti PD-1.

Conclusions We identify a consequence of CX3CR1 in terminal $\mathrm{T}$ cell exhaustion, and our work can offer strategies to increase responses to anti PD-1.

Ethics Approval Animal experiments were performed as per the IACUC regulations at the Dana Farber cancer Institute, and the MD Anderson Cancer Center

http://dx.doi.org/10.1136/jitc-2021-SITC2021.229 